\title{
Evidencias del impacto de las TIC en la productividad de la empresa. ¿Fin de la «paradoja de la productividad»?*
}

\author{
Margarita Billón Currás \\ Dpto. de Estructura Económica y Economía del Desarrollo \\ Universidad Autónoma de Madrid \\ margarita.billon@uam.es

\section{Fernando Lera López} \\ Dpto. de Economía \\ Universidad Pública de Navarra \\ lera@unavarra.es

\section{Salvador Ortiz Serrano} \\ Dpto. de Economía Aplicada \\ Universidad Autónoma de Madrid \\ salvador.ortiz@uam.es
}

\begin{abstract}
RESUMEN
En el presente artículo se efectúa una revisión y análisis de la evidencia empírica disponible con el fin de responder a la pregunta de si la «paradoja de la productividad» a nivel de empresa ha dejado de ser tal y en qué condiciones. A partir del marco teórico de la función de producción, se clasifica la literatura en torno a varias

* Los autores agradecen la ayuda recibida del Departamento de Educación del Gobierno de Navarra en el marco del proyecto de investigación «Gestión del ecosistema informativo de las TIC en la empresa industrial navarra» así como los valiosos comentarios efectuados por D. José Luis Zofío, profesor de la Universidad Autónoma de Madrid, y por varios evaluadores anónimos.
\end{abstract}


proposiciones; se revisan y comparan resultados, enfoques metodológicos, técnicas y muestras utilizados. A pesar de la existencia de múltiples sesgos, problemas de medición y factores condicionantes y complementarios, la evidencia existente a nivel de empresa apunta a que la paradoja de la productividad a nivel microeconómico parece haberse superado.

Palabras clave: Tecnologías de la Información y la Comunicación (TIC), productividad, cambio organizacional.

\begin{abstract}
This paper offers a review and an analysis of the available empirical evidence regarding the relationship between ICT and productivity at the firm level. Our aim is to answer the question to what extent and under what sort of conditions, the paradox at the firm level has been refuted. From the theoretical framework of the production function, the literature is classified around various propositions. Results, methodological approaches, techniques and samples are reviewed and compared. Despite the existence of many biases as well as measurement problems and conditioning factors, we conclude that the productivity paradox at microeconomic level seems to be refuted.
\end{abstract}

Keywords: Information and Communication Technologies (ICT), productivity, organisational change.

JEL: O33, D24.

\title{
1. INTRODUCCIÓN
}

La conocida frase acuñada por Robert Solow en 1987 «La era de los ordenadores puede verse en todas partes excepto en las estadísticas de productividad», supuso el origen del debate en torno a la «paradoja de la productividad», reflejo del hecho de que el aumento de las inversiones en Tecnologías de la Información y la Comunicación (TIC) no se reflejaba en los crecimientos de productividad esperados. La favorable evolución de la productividad laboral y de la productividad total de los factores de Estados Unidos y de algunos países de la OCDE en la segunda mitad de los 90, inició un debate en torno a si dicha evolución significaba el final de la paradoja. Así, en los últimos quince años diversos trabajos se han centrado en determinar el impacto de las TIC en el output y en el crecimiento de la productividad del trabajo a nivel 
agregado en Estados Unidos y algunos países europeos (Gordon, 2000; Oliner y Sichel, 2000; Jorgenson, 2001; Colecchia y Schreyer, 2002; Daveri, 2002; Stiroh, 2002; Timmer y van Ark, 2005, entre otros) tratando de aportar evidencia con la que refutar o corroborar la paradoja. A pesar de una falta de consenso en los primeros estudios y de importantes problemas de medición, la mayoría parece confirmar una relación positiva entre crecimiento de la productividad e inversiones en TIC a nivel agregado. Algunos autores sostienen, sin embargo, que los efectos sobre la productividad se han limitado a ciertos sectores manufactureros e industrias productoras de TIC (Gordon, 2000); mientras que otros (Nordhaus, 2001) demuestran que es el uso de las TIC en el resto de ramas productivas lo que explica el crecimiento de la productividad del trabajo. En el caso español, en cambio, la acumulación de capital TIC ha venido acompañada de una desaceleración del crecimiento de la productividad agregada del trabajo en la segunda mitad de los 90, aunque se ha registrado una aceleración a partir del 2000 (Mas y Quesada, 2005a, 2005b; Pilat, 2005; Estrada y otros, 2006). En varios estudios referidos al período 1985-2002, Mas y Quesada (2005a, 2005b) demuestran que los efectos de la inversión en TIC no se reflejan aún en el crecimiento de la productividad total de los factores. Sin embargo, las TIC contribuyeron anualmente al crecimiento de la productividad laboral en un 65,74\%. Los sectores intensivos en TIC han sido los principales responsables de dicha evolución, también en el período 2000-2004 (Mas y Quesada, 2006).

Aún cuando la afirmación de Solow hacía referencia a la paradoja a nivel agregado y ésta se manifestó especialmente en el período 1985-1995, desde la segunda mitad de los 90 y hasta la actualidad han ido proliferando las investigaciones orientadas a obtener evidencia de las relaciones entre inversión en TIC y crecimiento de la productividad y otras variables a nivel microeconómico. Las empresas son uno de los principales actores en la inversión en estas tecnologías. La posibilidad de cuantificar los retornos de dichas inversiones, de analizar sus efectos económicos y las condiciones en que impactan en la productividad, se inscribe dentro de lo que algunos han denominado el estudio de la segunda paradoja (Dehning y Richardson, 2002) y es uno de los ámbitos de mayor interés en la actualidad tanto para directivos como para investigadores (Mahmood y Szewczak, 1998). Aumentar nuestro conocimiento sobre lo que sucede en el interior de la «caja negra» de las compañías puede contribuir a explicar la naturaleza de los efectos de las inversiones en TIC sobre la productividad (Brynjolfsson y Hitt, 1998, 2003) y justifica la abundante bibliografía sobre este tema. En el caso particular de España, el estudio del comportamiento de las empresas en materia de inversiones en TIC y sus efectos sobre la productividad adquiere gran relevancia dado el necesario crecimiento de la productividad del país. 
La literatura ha puesto de manifiesto sin embargo que la evidencia de los efectos sobre la productividad y otras variables empresariales es a veces contradictoria y que el conocimiento de dichos efectos, si bien ha avanzado considerablemente, es aún limitado (Brynjolfsson y Yang, 1996; Hitt y Brynjolfsson, 1996; Mahmood y Szewczak, 1998; Dehning y Richardson, 2002; Kohli y Devaraj, 2003; Brynson y Ko, 2004; Oz, 2005).

Aparte de las ya citadas, existen otras razones que justifican el interés del estudio de este fenómeno a nivel microeconómico. El ámbito de análisis a nivel de empresa ofrece una mayor disponibilidad de datos frente a los ámbitos industrial y agregado (Stiroh, 2002), especialmente a partir del momento en que se empieza a disponer de datos de panel de inputs y outputs para un gran número de empresas, lo que favorece la utilización de técnicas econométricas que permiten analizar la contribución de las inversiones en TIC al crecimiento de la productividad total de los factores (Brynjolfsson y Hitt, 2003; Oz, 2005). Además, las investigaciones a nivel de empresa presentan cierto tipo de ventajas a la hora de analizar factores que no pueden ser captados adecuadamente a través de enfoques de medición tradicionales, tales como las inversiones organizacionales intangibles o las innovaciones de productos y servicios, y en general, a la hora de estimar el valor intangible asociado a la inversiones en TIC (Brynjolfsson y Hitt, 1998).

Todo lo anterior justifica el interés por efectuar una revisión de la literatura de los estudios a nivel de empresa que contribuya a clarificar si la evidencia recabada corrobora o refuta la paradoja de la productividad y en qué condiciones. Por otra parte, la gran variedad de efectos y vías por las cuáles estas tecnologías pueden impactar en la productividad y en su crecimiento, hacen conveniente la organización, sistematización y análisis de dicha evidencia en torno al marco conceptual existente, de forma que la revisión pueda ser relevante y útil para futuros trabajos de investigación.

La mayoría de las revisiones previas de la literatura sobre los impactos de las TIC en la productividad abarca conjuntamente tres ámbitos de análisis: nivel agregado, industria y empresarial. Los objetivos de dichas revisiones cubren el análisis de la contribución de las inversiones en TIC al excedente del consumidor y al crecimiento económico (Brynjolfsson y Yang, 1996); el análisis de los factores que explican el crecimiento de la productividad según los estudios que utilizan datos longitudinales (Bartelsman y Doms, 2000); el examen de las diferencias en las especificaciones de los modelos y las técnicas econométricas empleadas (Stiroh, 2002); la revisión crítica en los tres niveles (Dedrick y otros, 2003) o la elaboración de un meta-análisis de las variables estructurales recogidas en la investigación empírica a nivel de empresa (Kohli y Devaraj, 2003). En España, algunos estudios realizan interesantes revisiones a nivel de empresa como marco en el que situar la evidencia empírica obtenida (Gargallo y Galve, 2004; López Sánchez, 2004; Fuentelsanz y otros, 2005). 
El objetivo de este artículo es efectuar una revisión de la evidencia empírica disponible sobre los efectos de las TIC en la productividad, referida de manera particular al nivel de empresa e incluyendo la evidencia existente para el caso español, con el fin de responder a la pregunta: ¿en qué condiciones se ha refutado (o no) la paradoja de la productividad a nivel empresarial? La revisión se ha estructurado siguiendo un enfoque centrado en los conceptos (Webster y Watson, 2002). Así, partiendo del marco teórico de la función de producción y del marco conceptual que se deriva de la evidencia disponible sobre los impactos de las TIC en la productividad, los estudios se han categorizado en torno a dos proposiciones. La primera de ellas refleja el «imperativo tecnológico» (las inversiones en TIC por si solas explicarían el crecimiento de la productividad). La segunda incluye el papel de los cambios organizacionales e inversiones complementarias y su interacción con las TIC a la hora de determinar los factores que explican el crecimiento de la productividad (Miller y Rice, 1967; Francalanci y Galal, 1998; Gargallo y Galve, 2004). La clasificación de los trabajos considera también la existencia de distintos tipos de impactos de las TIC sobre el crecimiento de la productividad: directos e indirectos (Lehr y Lichtenberg, 1999; Mateucci y otros, 2005). El análisis de los trabajos incluye una comparación de las principales metodologías, técnicas, muestras, variables y resultados obtenidos. A partir de dicho análisis se detectan posibles problemas de medición, de especificación de los modelos, factores condicionantes de los resultados y futuras áreas de investigación.

El ámbito de revisión abarca estudios que analizan el impacto de las TIC sobre la productividad y en particular los referidos especialmente a los impactos derivados de la inversión y el uso de ordenadores, por ser estas tecnologías sobre las que existe una mayor evidencia empírica. No se han considerado otros estudios que analizan exclusivamente otros impactos y relaciones de las TIC en la empresa ni otro tipo de tecnologías.

La selección bibliográfica se ha realizado, siguiendo a Brynjolfsson y Yang (1996), Dedrick y otros (2003) y Kohli y Devaraj (2003) por búsqueda por palabras clave en revistas de impacto (Journal Citation Reports) en áreas de Economía, Organización de Empresas y en Sistemas de Información, dado el carácter interdisciplinar del tema. En la selección final de estos artículos se ha considerado a su vez el número de citas que de dichos artículos aparecen en otras revistas de impacto ${ }^{1}$. Se han con-

${ }^{1}$ Consultado el número de veces en que aparece citado cada artículo en otras revistas de impacto según el Social Sciences Citation Index, se han seleccionado aquellas que recogen un número de citas más elevado. 
sultado las bases de datos ABI Inform, Econlit, Bussines Source Elite y Current Contents. En el caso de los trabajos referidos a España, se han consultado las bases ISOC Dialnet y Compludoc. Se han revisado a su vez las referencias citadas en los artículos identificados previamente así como las referencias de aquellos artículos publicados posteriormente que incluyen citas de los artículos «clave» identificados en las etapas anteriores. También se han incluido investigaciones citadas por otras revisiones previas de la literatura ya mencionadas, así como otro tipo de estudios (artículos que no aparecen publicados en revistas de impacto, documentos, libros o capítulos de libros) que por su alto rigor metodológico y académico y dada su amplia difusión y el reconocido prestigio de sus autores, son obligada referencia en este área de estudio. Por último, se han considerado también documentos representativos elaborados por organismos internacionales como la OCDE. El período de referencia analizado abarca desde finales de los años 80 hasta el año 2006.

En la siguiente sección se recoge una breve aproximación al marco conceptual relativo a las vías por las que las TIC impactan en la productividad de la empresa. En el epígrafe 3 se analiza la evidencia empírica categorizada a partir del marco conceptual establecido. En el último apartado se presentan las principales conclusiones.

\section{Marco conceptual}

De los distintos enfoques utilizados para determinar los impactos de las TIC sobre la productividad de la empresa, la inmensa mayoría de los trabajos analizados utiliza la teoría de la producción. La función de producción relaciona cantidades de factores de producción empleados (inputs) con niveles de producto obtenidos (outputs) y con un estado dado de la tecnología. La función de producción más utilizada en los estudios analizados es la función Cobb-Douglas:

$$
Y=A K^{\alpha} L^{\beta}
$$

donde el output (Y) es una función exponencial de los inputs de capital $(\mathrm{K})$ y trabajo (L) multiplicados por un parámetro (A) que representa el nivel de la tecnología, siendo $\alpha$ y $\beta$ los coeficientes que representan las elasticidades output de cada uno de los inputs.

Siguiendo la metodología de la contabilidad del crecimiento de Solow, el crecimiento del output se puede descomponer en la suma de las tasas de crecimiento de cada uno de los factores de producción, multiplicadas cada una por la participación 
de su renta en la renta total de los factores (GAPTEL, 2004). La parte del crecimiento del output no explicada por la participación en el crecimiento de los factores de producción se calcula de forma residual y se conoce como Productividad Total de los Factores (PTF), también denominado Residuo de Solow.

La productividad de un factor se puede definir como la cantidad de output por unidad de input. Las mediciones de productividad más frecuentes son las medidas de la productividad del trabajo y del capital y las medidas de la PTF basadas en el valor añadido. En la función:

$$
A=\frac{Y}{K^{\alpha} L^{\beta}}=P T F
$$

la PTF representa el progreso tecnológico. En la actualidad sabemos que el residuo incluye también otros factores no tecnológicos de difícil medición entre los que se pueden citar la calidad de los inputs, en especial la calidad del trabajo, y otras variables como la inversión en innovación, errores de medición en las variables, externalidades, imperfecciones en los mercados, etc. (GAPTEL, 2004; OCDE, 2004; Pilat, 2004; Ontiveros y otros, 2004; Sanaú y otros, 2006). Las diversas medidas de productividad están a su vez interrelacionadas entre sí y las distintas relaciones entre ellas se suelen determinar a través de distintas técnicas, entre ellas, las econométricas que se aplican para estimar los parámetros de la función de producción y obtener así medidas directas del crecimiento de la productividad (OCDE, 2001).

El gráfico 1 nos ofrece una visión analítica del papel de las TIC en la función de producción. La introducción de las TIC en la empresa como un input más, por ejemplo a través de la inversión en ordenadores, puede transformar la cantidad y la calidad de los factores de producción. En la mayoría de los trabajos revisados las inversiones en TIC se definen como el valor del stock de las inversiones en ordenadores incluyendo la depreciación de las inversiones previas. En el citado gráfico aparecen el trabajo y el capital descompuestos en trabajo cualificado/trabajo no cualificado y capital TIC/capital no TIC. 
Gráfico 1. Impacto de las TIC en el proceso productivo

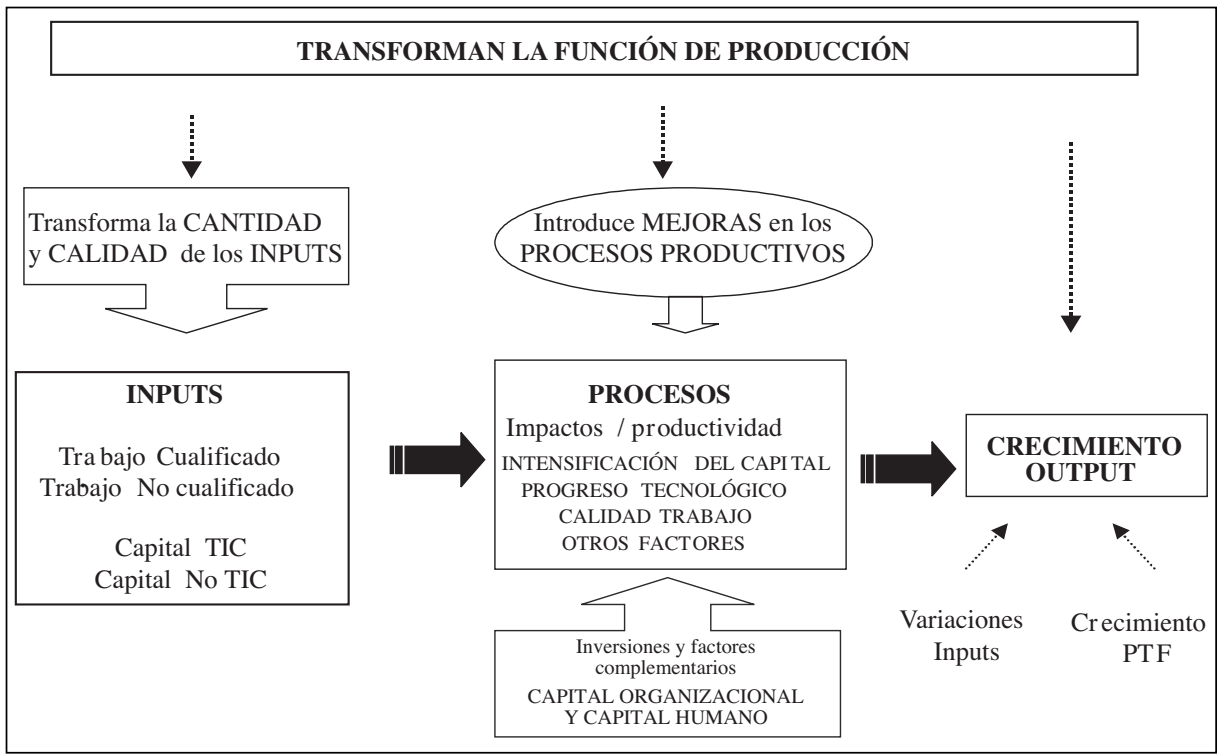

Fuente: Elaboración propia a partir de Dedrick y otros (2003).

Al aumentar las inversiones en ordenadores, el capital TIC cobra una mayor importancia en el stock de capital de las empresas, lo que se produce a la vez que los ordenadores van aumentando su capacidad para procesar información. Por otra parte, el capital no TIC se ve afectado de manera creciente por el capital TIC, por ejemplo, a través del impacto de los componentes electrónicos o del software informático (Greenan y otros, 2001). Las TIC también impactan en el input trabajo. Los ordenadores pueden contribuir al crecimiento de la productividad aparente del trabajo a través de la intensificación del capital (capital deepening) o aumento del capital por trabajador. Además, una utilización adecuada del capital TIC en la empresa requiere cambios en el papel que juega el factor trabajo, lo que puede hacer aflorar nuevas necesidades de cualificación y provocar cambios en la composición de la mano de obra y en su remuneración a favor de los trabajadores que utilizan las TIC.

Por otro lado, los inputs se transforman en outputs a través del proceso productivo. Las TIC contribuyen a introducir cambios en dichos procesos y por esta vía pueden contribuir a incrementar la PTF. Se puede distinguir entre impactos directos e indirectos de las TIC sobre la productividad (Matteucci y otros, 2005). El impacto directo se relaciona con el efecto de la inversión en TIC en términos de intensificación del capital e incluye la automatización o sustitución de trabajo por capital y el 
aumento de capital TIC por trabajador, lo que se puede traducir en un incremento de la productividad del trabajo. Los impactos indirectos de las TIC son más sutiles y difíciles de cuantificar y se manifiestan en transformaciones que tienen lugar en el proceso productivo y en los impactos en los sistemas de información (Gilchrist y otros, 2001).

Los impactos indirectos son el resultado de las especiales características de las TIC como «general purpose technologies» (Bresnahan y Trajtenberg, 1995) gracias a las cuales se pueden favorecer incrementos de productividad a través, no sólo de la reducción de costes sino, sobre todo, a través de mejoras en la calidad y de los aspectos intangibles de productos y procesos (Brynjolfsson y Hitt, 2000; Brynjolfsson y otros, 2002). Parte de la literatura existente ha destacado el papel de las TIC en la coordinación dentro de la empresa, pues éstas facilitan una utilización más eficiente de la información, lo que a su vez permite una toma de decisiones también más eficiente (Brynjolfsson y Hitt, 2000; Dewett y Jones, 2001; Gilchrist y otros, 2001).

Junto a la literatura que defiende que las inversiones en TIC son el principal factor que explican el crecimiento de la productividad (imperativo tecnológico) otras corrientes teóricas, como la Teoría de Recursos y Capacidades, la Teoría de las Complementariedades o el enfoque sociotecnológico (Gargallo y Galve, 2004) sostienen que existen factores complementarios, tales como los cambios organizacionales, que interactúan con las TIC a la hora de explicar dicho crecimiento (Milgrom y Roberts, 1990; Francalanci y Galal, 1998; Arnal y otros, 2001; Bertschek y Kaiser, 2004; Hollenstein, 2004). Dichos cambios organizativos e innovaciones complementarias podrían moderar o potenciar los efectos de las TIC (Brynjolfsson y Hitt, 2000; Bresnahan y otros, 2002; Melville y otros, 2004; Ontiveros y otros, 2004).

A través de los efectos indirectos que favorecen las transformaciones en el proceso productivo y en la toma de decisiones, las TIC, al venir asociadas a otros cambios tales como los organizacionales, pueden incrementar la PTF (Brynjolfsson y Hitt, 1998, 2000, 2003; Lehr y Lichtenberg, 1999; Brynjolfsson y otros, 2002; Dedrick y otros, 2003). A su vez, como consecuencia tanto de los efectos directos como indirectos se puede registrar un incremento de la productividad del trabajo. Ésta puede aumentar por tanto, por la intensificación del capital y por el incremento de la PTF. Como resultado de lo anterior puede registrarse a su vez un incremento de la producción que puede deberse a la variación en la utilización de los factores productivos y/o a los cambios en la PTF.

A partir de este marco conceptual en el siguiente epígrafe se organiza la evidencia empírica seleccionada en torno a los impactos de las inversiones y uso de las TIC sobre la productividad. 


\section{REVISIÓN DE LA EVIDENCIA EMPÍRICA}

La tabla 1 muestra una selección de los estudios internacionales y españoles revisados que cubre el período 1976-2004. En ella aparece información sobre la muestra seleccionada (período, número de empresas, país, sector), modelo especificado, métodos econométricos empleados, variables incluidas (dependientes y explicativas) y principales resultados. La evidencia empírica se ha organizado en torno a dos proposiciones, considerando por una parte los estudios que investigan los impactos de las inversiones en TIC en el output y el incremento de la productividad del factor trabajo, y por otra, estudios que consideran las relaciones entre las inversiones en TIC (ordenadores) y otros factores no tecnológicos como los cambios organizacionales y del capital humano.

\subsection{Inversiones en TIC, output y productividad del trabajo}

Proposición 1: Las inversiones en TIC (ordenadores) incrementan el output y la productividad del factor trabajo.

Desde finales de los años 80 la literatura sobre los efectos de las inversiones en TIC, y en particular de la inversión en equipo informático, gira en torno a lo que hemos denominado efectos «directos», a través de la intensificación del capital en la empresa y por esta vía impactan en el crecimiento del output y la productividad del trabajo.

Varios de los estudios de finales de los 80 y principios de los 90 a nivel de empresa no encuentran evidencia de que las inversiones en TIC conduzcan a variaciones en el output ni en la productividad. Los trabajos de Strassmann (1990), Roach (1991), Loveman (1994) y Barua y otros (1995) son ejemplos de ello. El hecho de que estos primeros trabajos no encontraran una relación entre ambas variables puede deberse a múltiples razones, entre ellas el tamaño de las muestras y la calidad de los datos, en especial los problemas de medición de los costes de las inversiones en equipo informático (Brynjolfsson y Yang, 1996) y posiblemente también al hecho de que las empresas no alcanzaran niveles de inversión en TIC como los que se han ido registrando posteriormente (Dedrick y otros, 2003; Fuentelsaz y otros, 2005) y que han ido reflejándose en incrementos de productividad a medida que las empresas se han ido adaptando a dichas tecnologías. 


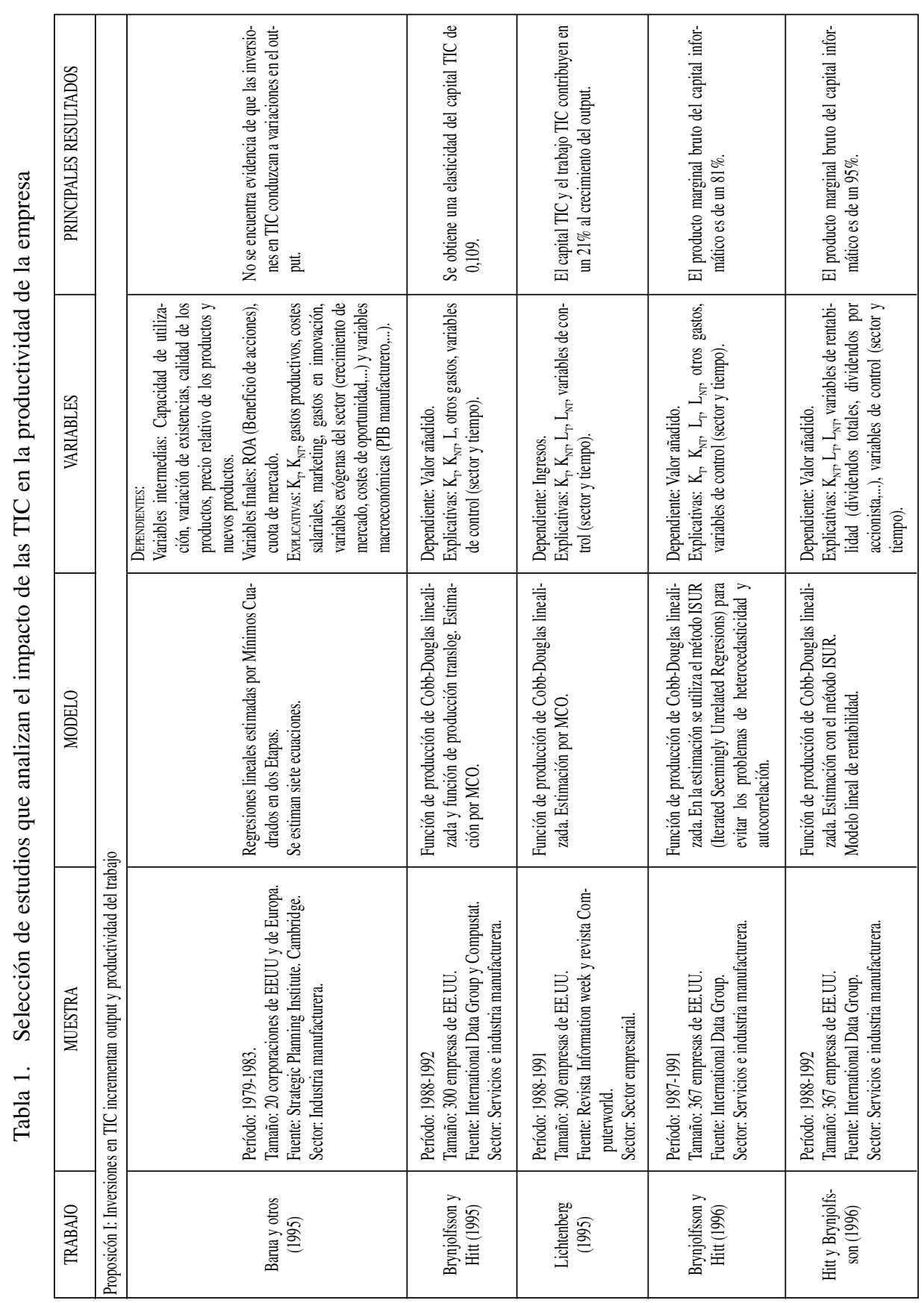




\begin{tabular}{|c|c|c|c|c|c|c|}
\hline 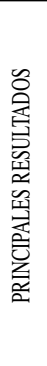 & 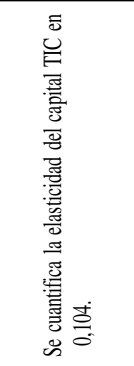 & 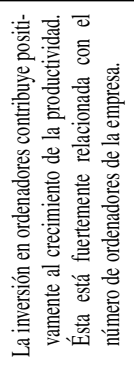 & 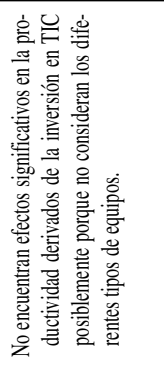 & 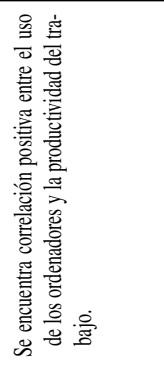 & 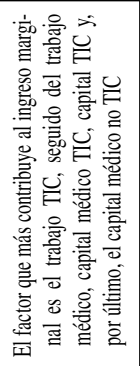 & 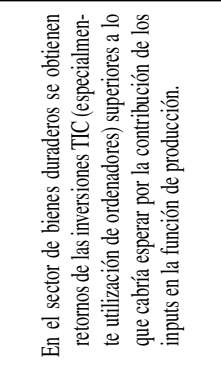 \\
\hline $\begin{array}{l}\text { 留 } \\
\text { 密 } \\
\text { 变 }\end{array}$ & 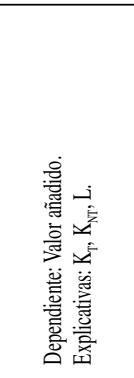 & 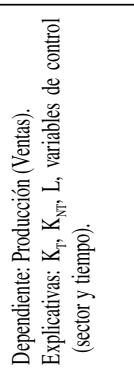 & 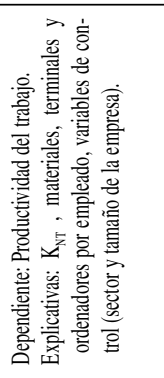 & 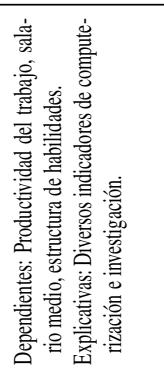 & 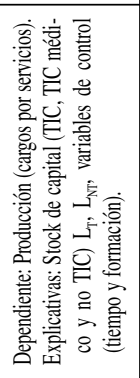 & 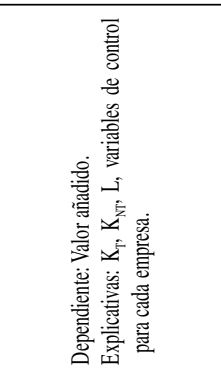 \\
\hline 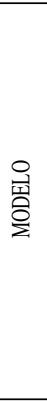 & 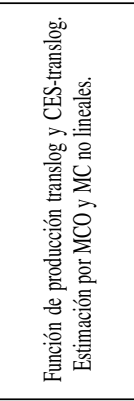 & 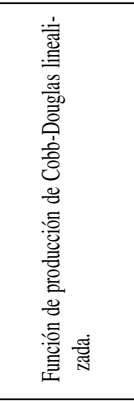 & 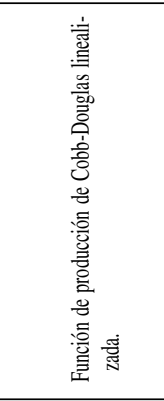 & 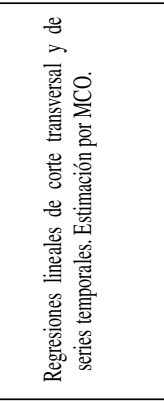 & 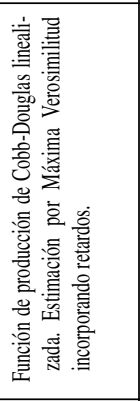 & 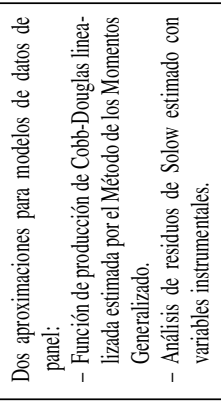 \\
\hline $\begin{array}{l}\text { 焉 } \\
\text { 䍚 }\end{array}$ & 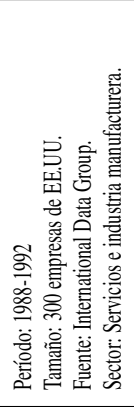 & 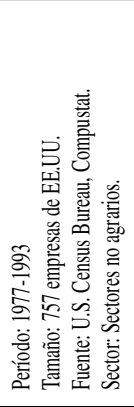 & 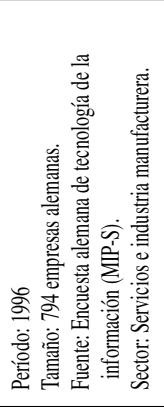 & 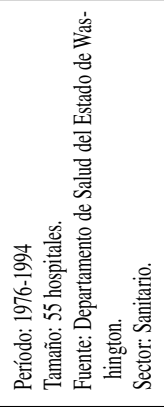 & 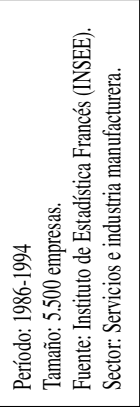 & 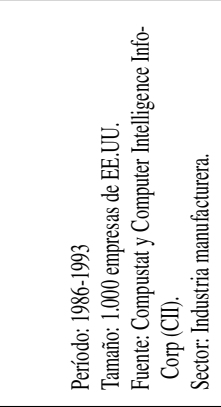 \\
\hline 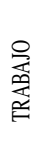 & 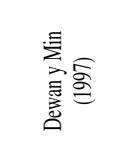 & 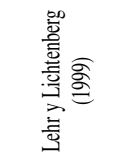 & 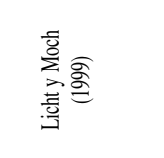 & 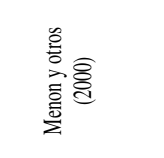 & 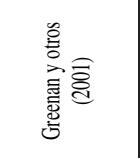 & $\begin{array}{l}\text { 号 } \\
\text { 总 } \\
\text { 总 } \\
\text { 总 }\end{array}$ \\
\hline
\end{tabular}




\begin{tabular}{|c|c|c|c|c|c|c|}
\hline 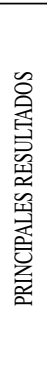 & 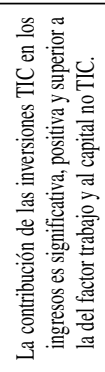 & 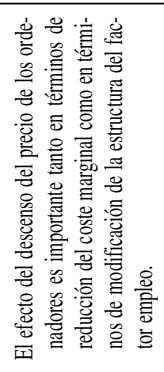 & 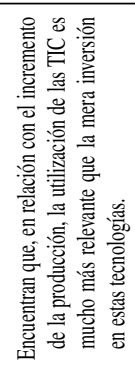 & 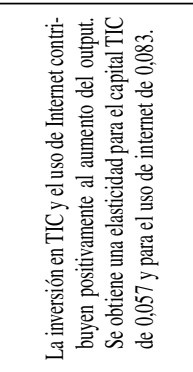 & 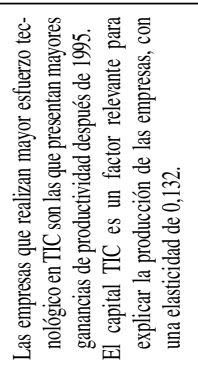 & 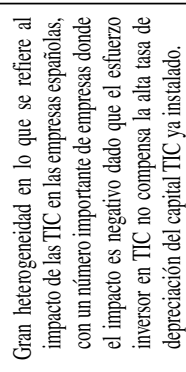 \\
\hline $\begin{array}{l}\text { 留 } \\
\text { 产 } \\
\text { 恙 }\end{array}$ & 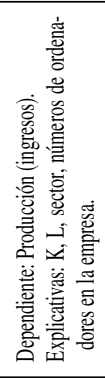 & 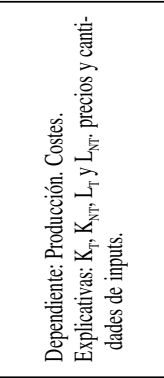 & 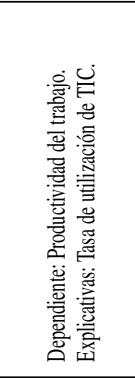 & 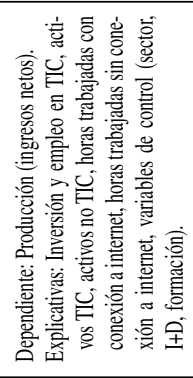 & 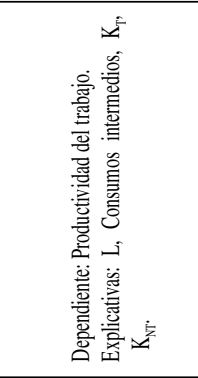 & 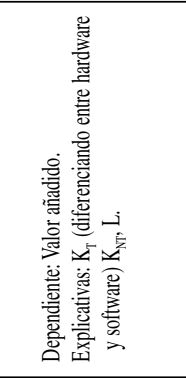 \\
\hline 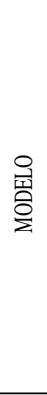 & 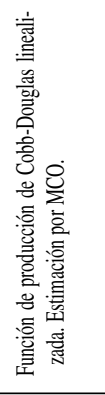 & 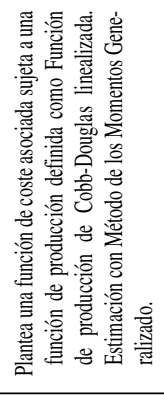 & 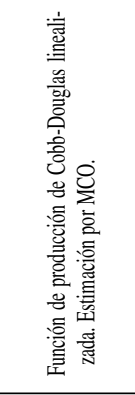 & 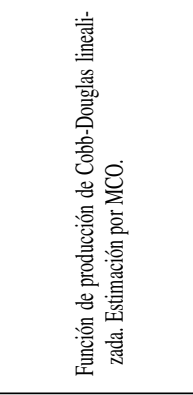 & 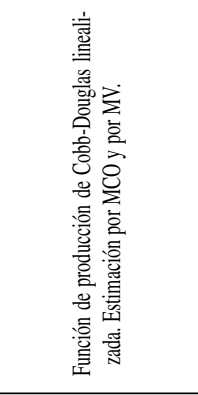 & 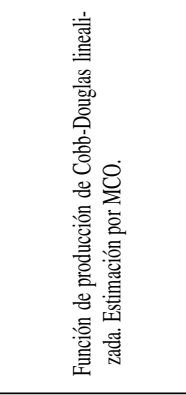 \\
\hline 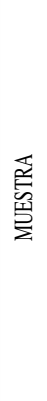 & 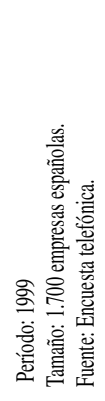 & 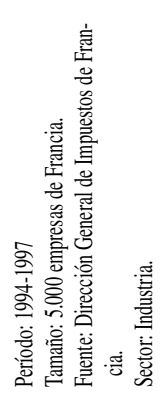 & 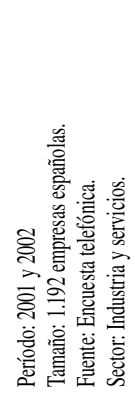 & 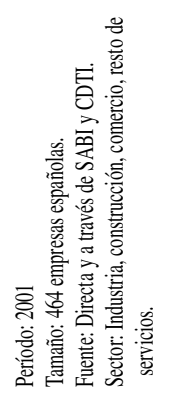 & 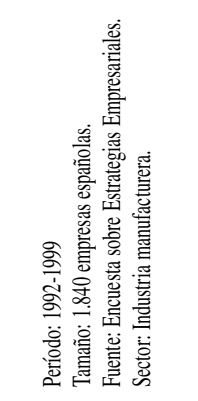 & 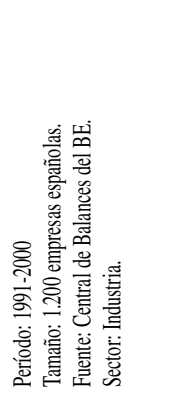 \\
\hline 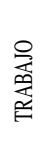 & $\begin{array}{l}\widehat{\bar{d}} \\
\text { 产 } \\
\overline{0}\end{array}$ & 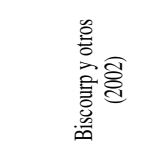 & 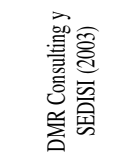 & 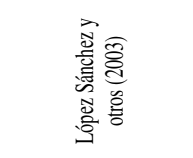 & 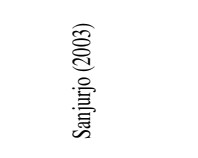 & 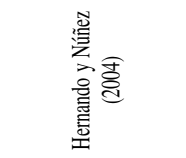 \\
\hline
\end{tabular}




\begin{tabular}{|c|c|c|c|c|c|c|c|}
\hline 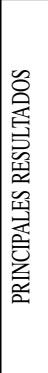 & & 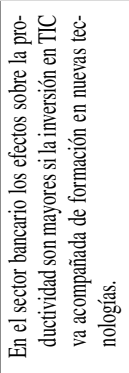 & 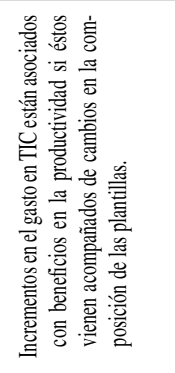 & 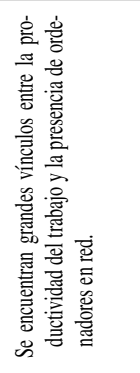 & 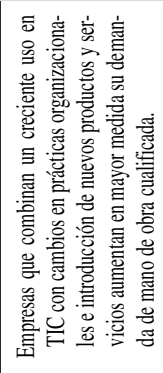 & 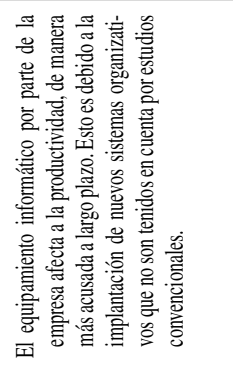 & 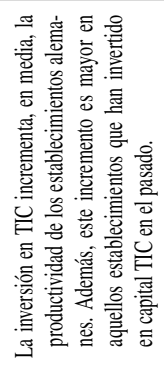 \\
\hline 凅 & & 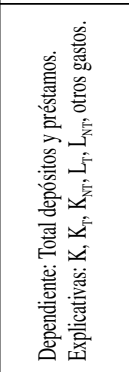 & 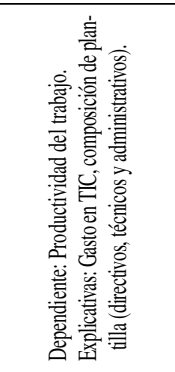 & 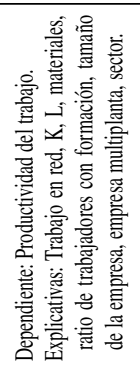 & 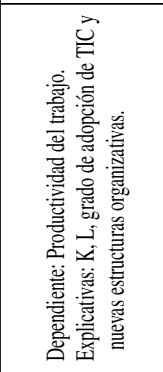 & 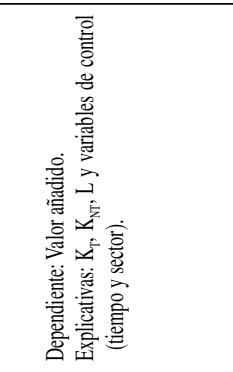 & 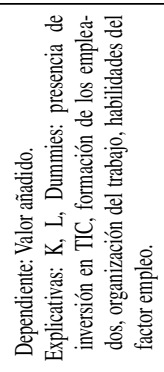 \\
\hline 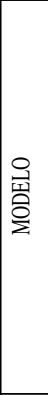 & 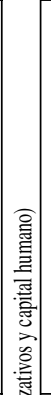 & 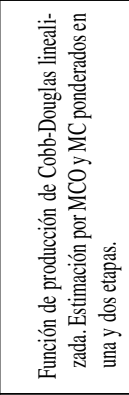 & 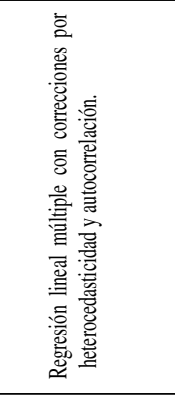 & 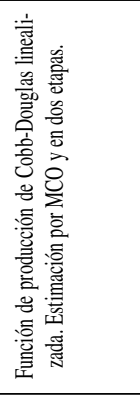 & 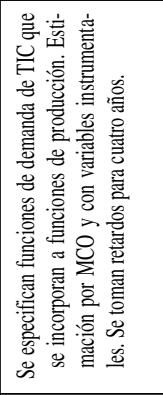 & 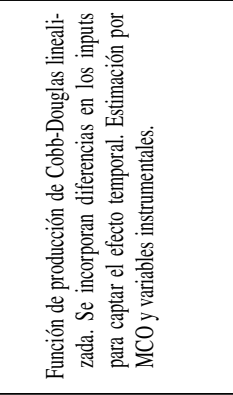 & 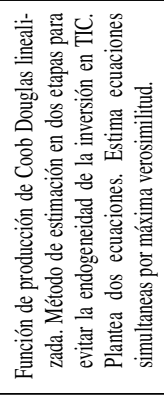 \\
\hline $\begin{array}{l}\text { 总 } \\
\text { 窟 } \\
\text { 竞 }\end{array}$ & 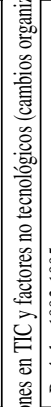 & 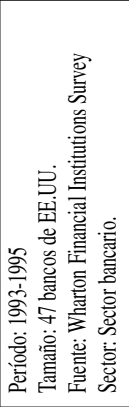 & 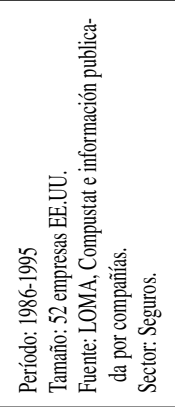 & 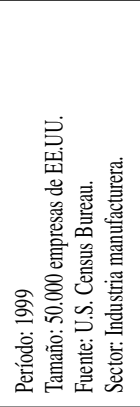 & 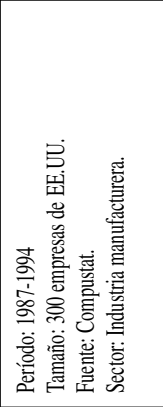 & 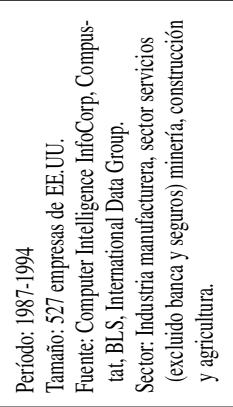 & 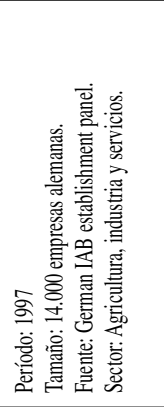 \\
\hline 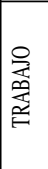 & 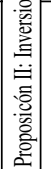 & 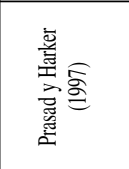 & 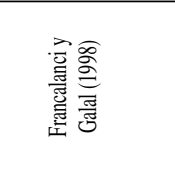 & 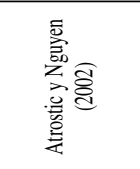 & 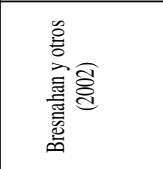 & 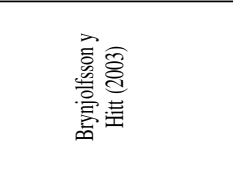 & 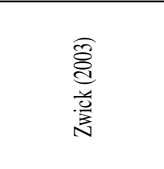 \\
\hline
\end{tabular}


EVIDENCIAS DEL IMPACTO DE LAS TIC EN LA PRODUCTIVIDAD DE LA EMPRESA

¿FIN DE LA «PARADOJA DE LA PRODUCTIVIDAD»?

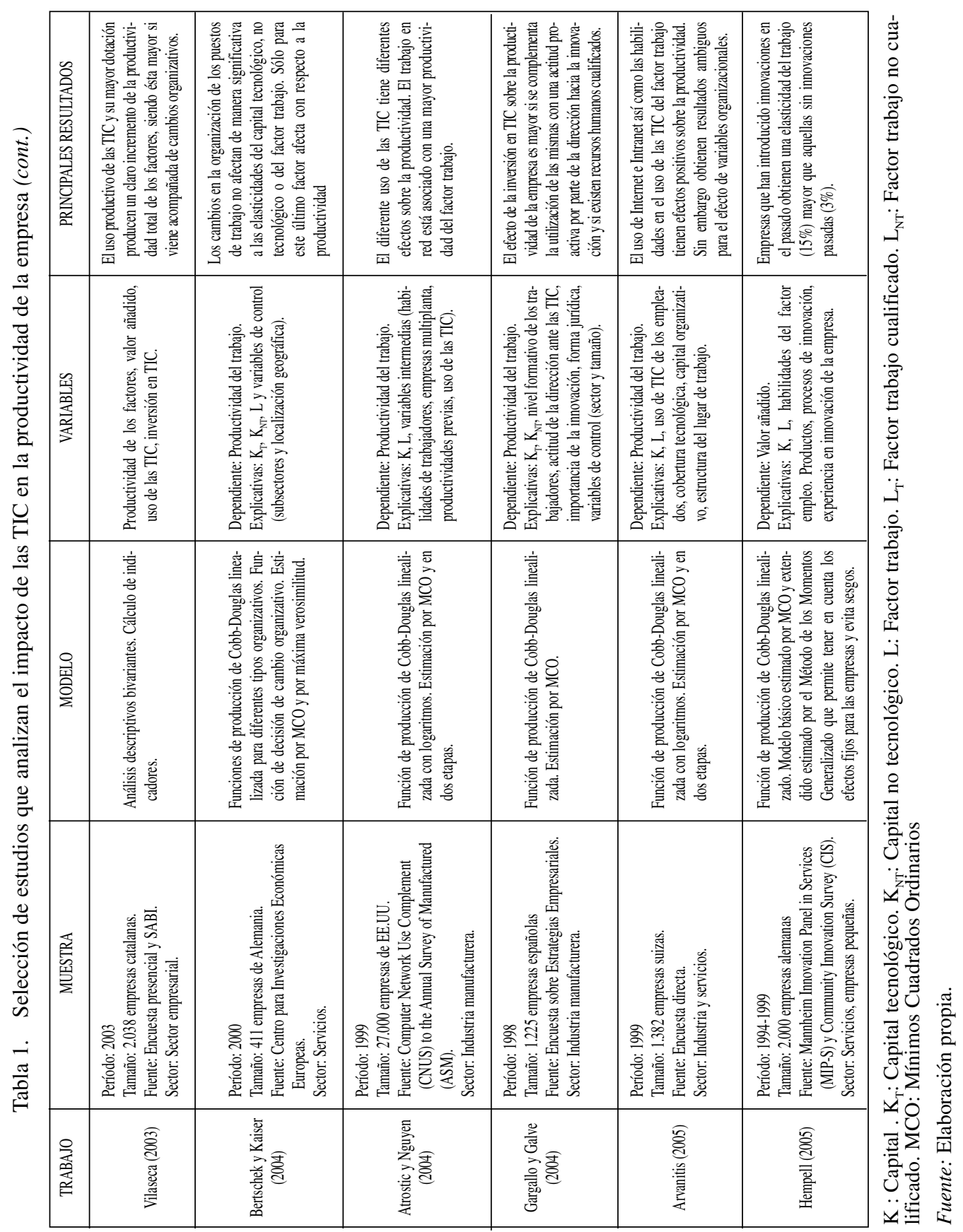


A medida que avanza la década de los 90 , muchos estudios se centran en demostrar que las inversiones en TIC contribuyen de manera significativa al crecimiento del output. Diversos autores analizan conjuntamente la contribución al output de las inversiones en capital TIC y del trabajo relacionado con las TIC.

El modelo considerado inicialmente por gran parte de la literatura consiste en la estimación mediante Mínimos Cuadrados Ordinarios (MCO) de una función de producción de Cobb-Douglas linealizada mediante logaritmos. En esta función, el factor capital se divide entre capital TIC y capital no TIC para estudiar el impacto de la inversión del primero además de diversas variables de control (sector y tiempo fundamentalmente). Ante las limitaciones en la estimación que presentan distintas cuestiones como la heterogeneidad no observada, la autocorrelación o la endogeneidad de determinadas variables, en posteriores trabajos se utilizan otros métodos de estimación que pretenden solventarlos, como son el método ISUR para solucionar los problemas de heterogeneidad y autocorrelación, los mínimos cuadrados en dos etapas, la estimación por máxima verosimilitud, la utilización de variables instrumentales y el método de los momentos generalizados.

Pocos son los trabajos de la década de los noventa que no consideran este modelo básico. Cuando se considera este tipo de modelo se asume que las elasticidades output son constantes para cualquier vector de inputs y nivel de producción, además de que la elasticidad de sustitución de Allen para cualquier par de inputs es igual a 1. Otros autores (Dewman y Min, 1997) utilizan una función de producción CEStrasnslog, más flexible que la de Cobb-Douglas aunque de mayor dificultad tanto en la estimación como en la interpretación de variables. La función CES permite que la elasticidad de sustitución pueda ser cualquier número real y la función translog aumenta la flexibilidad permitiendo que las elasticidades sean distintas para diferentes empresas según el nivel de cada output, aunque complica su estimación así como la interpretación de los resultados. A pesar de sus limitaciones, la función de CobbDouglas es la más utilizada debido a la mayor facilidad de estimación y de interpretación.

Especialmente a partir de 1995, los estudios tienen en cuenta el efecto de las inversiones pasadas en el rendimiento presente de las empresas, a través de la inclusión de retardos en la especificación de los modelos. Igualmente, los trabajos van diferenciando el factor trabajo en trabajo cualificado y no cualificado para la utilización de las TIC.

En general, la evidencia existente demuestra la existencia de retornos de las inversiones en TIC superiores a lo que correspondería si atendemos a la participación de los inputs en la función de producción (los productos marginales exceden sus cos- 
tes directos). Ampliados sucesivamente la muestra de empresas y el período analizado, estos resultados referidos en su mayoría a Estados Unidos, se encuentran en trabajos tales como Brynjolfsson (1993), Brynjolfsson y Hitt (1995), Lichtenberg (1995), Hitt y Brynjolfsson (1996), Menon y otros (2000). Por ejemplo, Brynjolfsson y Hitt (1996) obtienen que el producto marginal bruto del capital informático es de un $81 \%$ por año para las 367 empresas de la muestra en el período 1987-1991, siendo al menos tan grande como el producto marginal de otros tipos de inversión en capital y siendo la contribución del trabajo TIC al crecimiento del output tan elevada como la del trabajo no TIC (Véase tabla 1).

Por lo que se refiere a España, no son muy numerosos los estudios a nivel de empresa que analizan el impacto de las TIC. Las carencias del sistema estadístico nacional provocan que las muestras utilizadas no abarquen gran número de años y que no procedan de operaciones estadísticas oficiales. Los métodos de estimación, modelos y resultados obtenidos son similares a los ya comentados ${ }^{2}$. Así, DMR Consulting y SEDISI (2003) demuestran que un $18 \%$ y un $11 \%$ del crecimiento de la productividad en los sectores del comercio y hostelería y la construcción, respectivamente, se explicaría por la inversión TIC. Dans (2001) en un trabajo referido a 1.700 PYMES obtiene un retorno de la inversión en ordenadores del 93,9\%, si bien el efecto no es lineal ya que éste aumenta cuanto menor es el número de PCs en la empresa. Hernando y Nuñez (2004) obtienen que el capital TIC explica cerca del $25 \%$ del crecimiento de la productividad del trabajo. Los autores encuentran gran heterogeneidad en lo que se refiere al impacto de las TIC en las empresas españolas, con un número importante de ellas en las que el impacto es negativo dado que el esfuerzo inversor en TIC no compensa la alta tasa de depreciación del capital TIC ya instalado. En la misma línea se ubica el trabajo de Sanjurjo (2003).

A lo largo del tiempo se ha ido constatando que para determinar el impacto de las TIC lo verdaderamente relevante es analizar no sólo la inversión TIC sino el uso de estas tecnologías. Diversos estudios han demostrado que un mayor uso de ordenadores en la empresa provoca incrementos en la productividad del trabajo (Lehr y Licthtenberg, 1999; Gilchrist y otros, 2001; Greenan y otros, 2001; Gretton y otros, 2002). Sin embargo, también es cierto que en el largo plazo, una vez se ha generalizado la inversión y el uso de los ordenadores dentro de la empresa, se pueden registrar ren-

${ }^{2}$ Un análisis sobre la medición de la productividad en España puede encontrarse en Uriel (2006). 
dimientos marginales decrecientes en el uso de las TIC. Este es uno de los resultados obtenidos para España por DMR Consulting (2003, 2004).

Las tecnologías informáticas son utilizadas por trabajadores más cualificados que, a su vez, van adquiriendo mayor capacidad de aprendizaje y se convierten en más productivos a medida que se incrementa su experiencia en el uso de las TIC (Entorf y Kramarz, 1998). Los cambios en la cualificación derivados de la introducción de las TIC se traducen en un incremento de salarios de los trabajadores más cualificados (Dewett y Jones, 2001; Bresnahan y otros, 2002; Autor y otros, 2003). En la primera mitad de los 90 distintos estudios encuentran una correlación positiva entre los trabajadores que utilizan ordenadores en su trabajo y los salarios más altos. De este modo, Krueger (1993) demuestra que la diferencia llega a ser entre un 10 y un 16\% de salarios más altos para estos trabajadores y Dunee y Schmitz (1995) y Autor y otros (1998) encuentran que las inversiones en ordenadores podrían explicar entre un 35 y un $50 \%$ del aumento de salarios de los trabajadores más cualificados.

Por otra parte, la evidencia disponible sugiere que se necesita tiempo para que las empresas se adapten a la introducción de las TIC y para que ello se refleje en retornos de dichas inversiones. Durante ese período de adaptación y aprendizaje, existen otros factores no tecnológicos tales como los cambios organizativos que pueden aparecer como potenciadores o limitadores de los efectos de las inversiones en TIC sobre la productividad. Doms y otros (1997) demuestran que en algunas plantas aumenta el nivel de cualificación de los trabajadores en el período previo y posterior de la adopción de las nuevas tecnologías. Esas plantas son altamente productivas tanto antes como después de la adopción de esas nuevas tecnologías. Los resultados indican que la adopción de nuevas tecnologías se produce con mayor probabilidad en plantas que tienen ya trabajadores cualificados, pero el hecho de adoptar las TIC no altera significativamente los salarios pagados o la estructura del empleo dentro de las plantas. Los autores lo atribuyen a que el ajuste de la mano de obra se realiza antes de la adopción de las nuevas tecnologías.

Desde la década de los noventa parte de la literatura se ha centrado en el estudio de los efectos indirectos de las inversiones en TIC sobre la productividad relacionándolos más ampliamente con cambios organizacionales e inversiones complementarias (Milgrom y Roberts, 1990). Ésta constituye la segunda proposición en torno a la que se ha clasificado la evidencia disponible y que abordamos a continuación. 


\subsection{Inversiones en TIC y otros factores no tecnológicos}

Proposición 2: Los impactos de las inversiones en TIC sobre la productividad se registran en conjunción con otros factores no tecnológicos (cambios organizativos y capital humano) y por esta vía afectan a la productividad del trabajo y a la productividad total de los factores.

Como tecnologías de coordinación las TIC impactan en la productividad al impulsar cambios en la estructura organizativa a través de mejoras en los flujos de información entre los trabajadores y la dirección, favoreciendo la creación de equipos de trabajo, la descentralización en la toma de decisiones, el achatamiento de las jerarquías, promoviendo cambios en la composición del trabajo a favor de los trabajadores más cualificados o animando las inversiones en formación (Arnal y otros, 2001; Bresnahan y otros, 2002; Brynjolfsson y Hitt 2003; Kohli y Devaraj, 2003; Bertschek y Kaiser, 2004; Hollenstein, 2004; Bayo y otros, 2007). A su vez, empresas en las que se han introducido cambios organizativos previos es probable que registren mayores inversiones en TIC y que ello a su vez afecte a los retornos obtenidos (Doms y otros, 1997; Brynjolfsson y Hitt, 2000; Black y Lynch, 2001).

En distintas aportaciones (Brynjolfsson y Hitt, 1998, 2000, 2003; Brynjolfsson y otros, 2002) se encuentran evidencias significativas de las relaciones entre las inversiones en TIC y el crecimiento de la PTF considerando el papel complementario que juegan los cambios organizacionales y las posibles diferencias en la medición de su impacto según nos refiramos al corto o al largo plazo. (Brynjolfsson y Hitt, 1998, 2000). Estos autores sostienen que los retornos en el corto plazo reflejan los efectos directos de la inversión en TIC, mientras que en el largo plazo representan los efectos de las TIC cuando se combinan con el cambio organizacional.

A partir del año 2000 muchos estudios incorporan variables organizacionales en los modelos, si bien los autores se enfrentan con la dificultad de medir los cambios organizativos. Son varias las alternativas que se proponen, casi todas encaminadas a la creación de variables ficticias que muestren la presencia o ausencia de una determinada característica organizacional en la empresa. Esta solución, aunque positiva, presenta la limitación de no poder matizar las diferencias de calidad en dichos cambios. Así, Arvanitis (2005) crea una serie de variables ficticias para observar la presencia de uso intensivo de equipos de trabajo, la rotación en el empleo y el descenso del número de niveles de gerencia, entre otras; Matteucci y otros (2005) introducen variables ficticias para observar la introducción de equipos 
de trabajo, la reducción de jerarquías y la introducción de grupos de trabajo autónomos.

A largo de la presente década un número creciente de estudios analiza la correlación entre las inversiones en TIC, cambios en la organización, especialmente en la organización del trabajo y la cualificación de los trabajadores, y sus impactos sobre la productividad. Francalanci y Galal (1998) en un estudio aplicado a las compañías de seguros demuestran que la introducción de las TIC viene acompañada de ganancias en productividad en empresas en las que aumenta el peso del componente directivo y se modifica la composición del trabajo a favor de una mano de obra más cualificada y cara.

Black y Lynch (1996, 2001, 2004) obtienen que existe correlación entre la introducción de nuevas prácticas en el lugar de trabajo, la creciente difusión del uso de los ordenadores y el crecimiento de la productividad en el sector manufacturero de Estados Unidos en el período 1993-1996. Cuanto mayor es la participación de los trabajadores en la toma de decisiones relacionadas con las mejoras en los procesos de producción asociadas a las TIC y mejor la relación entre aquéllos y los directivos de la empresa, mejores son los resultados obtenidos en términos de productividad del trabajo. Asimismo, cuanto más alto es el nivel educativo de los trabajadores y mayor la proporción de éstos y de directivos que utiliza ordenadores, más probable es que las empresas obtengan resultados por encima de la media y una productividad del trabajo más alta. Por último, estas autoras demuestran también que las plantas que introducen cambios en las prácticas laborales y en las que están presentes los sindicatos experimentan mayores impactos sobre la productividad.

Arvanitis (2005) investiga la influencia de las TIC, las nuevas prácticas en la organización del trabajo y la formación sobre la productividad de la empresa. Los resultados apuntan a que las empresas con una mayor participación de trabajadores cualificados obtienen mayor productividad de las TIC mientras que las nuevas prácticas organizacionales no parecen contribuir de manera significativa a una mayor productividad.

Bresnahan y otros (2002) hallan que aquellas empresas que combinan un creciente uso de las TIC con cambios en las prácticas organizacionales y la introducción de nuevos productos y servicios, incrementan en mayor medida su demanda de mano de obra cualificada. Estos autores hallan efectos interactivos dos a dos para las inversiones en TIC y los cambios organizativos por una parte y para las inversiones en TIC y la cualificación y formación por otra. Sin embargo, resulta difícil determinar el impacto de cada una de las tres variables de manera independiente.

En España, Gargallo y Galve (2004) que obtienen que la contribución de las TIC a la productividad es significativa y positiva — aunque menor que el capital no TIC- 
concluyen que las inversiones en TIC deben complementarse con una actitud proactiva hacia la innovación por parte de la dirección y con recursos humanos cualificados. En un amplio e interesante estudio, Vilaseca (2003) demuestra que el uso productivo de las TIC y su mayor dotación producen un claro incremento de la productividad total de los factores y que ésta aumenta con el cambio organizativo. En el estudio ya citado, DMR Consulting y SEDISI (2003) demuestran que las inversiones en TIC son condición necesaria pero no suficiente a la hora de explicar la productividad, destacándose la importancia de los cambios en los procesos de negocio como condición para que la empresa se adapte a estas tecnologías. Ontiveros y otros (2004) destacan la importancia de adoptar estructuras organizativas basadas en una mayor flexibilidad para poder utilizar las TIC como estrategia diferenciadora frente a la competencia. Pérez y otros (2006) demuestran una relación positiva entre la adopción de innovaciones organizativas, como el teletrabajo, y la adopción de TIC y la obtención de mejores resultados en las PYMES ${ }^{3}$.

\subsection{Sesgos y factores condicionantes}

La evidencia disponible, tanto en lo que respecta al papel determinante de las TIC en la productividad como a sus relaciones con los cambios organizacionales, presenta importantes sesgos y refleja la existencia de considerables problemas de medición que pueden condicionar los resultados comentados:

- Dificultad para medir el stock de capital TIC. Buena parte de los estudios revisados utilizan como variable para medir las inversiones en TIC, el valor de reposición del hardware, lo que puede estar subestimando las inversiones en ordenadores ya que no se consideran como tal inversiones en software, en formación u otros gastos relacionados con los ordenadores

- La medición tradicional de inputs y outputs no resulta adecuada para medir fuentes de valor no tradicionales. Existen deficiencias a la hora de capturar a través de los precios las mejoras de calidad o la mayor variedad de productos

${ }^{3}$ Numerosos estudios que evidencian los impactos de las TIC a nivel de empresa en el seno de los países de la OCDE pueden consultarse entre otros en OCDE (2001, 2003), Pilat (2004), Pilat y Wickoff (2005). 
y servicios. Estos problemas de medición se agudizan en el sector servicios, principal usuario de estas tecnologías ${ }^{4}$.

- El output es también función del capital intangible, de muy difícil medición. Además, las inversiones intangibles complementarias a las inversiones en TIC, especialmente en capital organizacional y humano, suelen manifestar su impacto a largo plazo, complicando la medición de sus efectos.

- Los problemas de medición citados pueden provocar heterogeneidad no observable (Zwick, 2003), lo que se puede traducir en sesgos e inconsistencias en los resultados. La heterogeneidad se puede producir tanto por no incluir variables relevantes en el modelo, como por no reflejar diferencias de calidad en estas variables. A ello hay que añadir la posible endogeneidad en las variables explicativas — por ejemplo, la decisión de inversión en TIC-, lo provocaría que las estimaciones estuvieran sesgadas. Una manera de solucionar esta limitación ha sido la utilización de ecuaciones simultáneas o de variables instrumentales (Barua y otros, 1995; Francalanci y Galal, 1998; Bresnahan y otros, 2002; Zwick, 2003).

- Las múltiples y complejas relaciones entre los diversos cambios organizativos y la implantación de las TIC dificulta la determinación de la dirección de la causalidad entre las variables organizacionales y las inversiones en TIC.

Por otra parte, resulta complicada la comparación de los resultados de los distintos trabajos, entre otras razones porque éstos pueden depender de las características de las muestras. Kohli y Devaraj (2003) encuentran que las relaciones más fuertes entre TIC y productividad aparecen en trabajos con número mayor de empresas y en aquellos que, además, usan información de carácter longitudinal.

El efecto de la inversión en TIC sobre la productividad también varía en función del sector analizado. Buena parte de los trabajos efectuados durante la década de los 80 estuvo referida al sector manufacturero por la falta de datos y por la dificultad de identificar adecuadamente el output del sector servicios. Los trabajos realizados durante la década de los 90, aunque utilizan en su mayor parte datos de EE.UU. y se

${ }^{4}$ Sobre las principales limitaciones para la estimación del stock de capital TIC puede consultarse Ontiveros y otros (2004). Llanos Matea (2006) ofrece evidencia para España de los problemas de medición por cambios de calidad de las TIC. 
refieren al sector manufacturero, incluyen ya otros sectores como servicios, banca, seguros, sanidad y se amplían a diversos países del área de la OCDE (OCDE, 2001, 2003; Pilat, 2004; Pilat y Wickoff, 2005). La evidencia demuestra que el efecto de la inversión en TIC varia considerablemente en función del sector (Gilchrist y otros, 2001; Gretton y otros, 2002; Devaraj y Kohli, 2003). Para España, Más y Quesada (2005a) obtienen que aquellos sectores más intensivos en TIC son los que mayores incrementos de productividad registran en el período 1985-2002. Por su parte López Sánchez (2004) demuestra que los mayores incrementos de productividad se registran en sectores con bajo grado de implantación de las TIC y siempre en función del tamaño de las empresas en las que se implanta dicha tecnología.

De hecho, el tamaño es una de las características de la empresa que más puede condicionar los resultados. El stock de inversión en el pasado, las decisiones sobre la realización de inversión en TIC, el tipo de tecnologías en las que se invierte y las decisiones en torno al trabajo TIC pueden diferir enormemente entre las pequeñas y grandes empresas. Lamentablemente, la mayor parte de los trabajos analizados aparecen sesgados hacia empresas de tamaño medio y grande. Una excepción es el trabajo de Vilaseca (2003) en el que se obtiene una relación significativa entre dimensión empresarial y la eficiencia en el uso de las TIC. En las microempresas el efecto es negativo por el coste relativo que implica la inversión en TIC y la necesidad de complementarlo con dotaciones de factor humano. No obstante, superado el impacto negativo de esta inversión y analizando el uso de las TIC, los incrementos de productividad son superiores a los que presentan las grandes empresas. Gimeno (2006) demuestra que el tamaño de la empresa española condiciona tanto la inversión como los gastos corrientes en TIC. Asimismo, el número de plantas o establecimientos condiciona positivamente la adopción de las TIC (Bayo y Lera, 2007).

La evidencia también demuestra que los impactos sobre la productividad dependen del tipo de tecnologías TIC. La productividad del trabajo es mayor cuando se utilizan redes ya que por sus especiales características contribuyen a generar efectos derrame y a difundir los impactos de las TIC (Bartlesman y otros, 1996; Goss, 2001; Atrostic y Nguyen, 2002; Baldwin y otros, 2004; Clayton y otros, 2004). En el caso español, López y otros (2003) demuestran que aumentar en una hora la utilización de Internet produce un incremento de un $4 \%$ en la cantidad producida, obteniéndose por cada euro adicional gastado en capital TIC un retorno de un $36 \%$. A su vez, la utilización de distintos tipos de TIC aumenta con el tamaño de la empresa y de las plantas. Las grandes empresas tienden a utilizar una combinación de redes tecnológicas, mientras que las pequeñas empresas suelen iniciarse con la inversión en ordenadores y en menor medida con Internet (OCDE, 2004). 
Otras características complementarias de la empresa tales como la estructura organizativa (Brynjolfsson y Hitt 1998; Dewan y Steven 1998; Dans, 2001), el aprendizaje y la cultura empresarial (Brynjolfsson y Hitt, 1995; Dewett y Jones, 2001) o la capacidad de innovación (Fuentelsaz y otros, 2005) pueden igualmente condicionar los impactos de las TIC. Esto justifica que ciertos trabajos introduzcan numerosas variables de control para capturar las características de los establecimientos. Así, por ejemplo, Kohli y Devaraj (2003) obtienen que en las empresas públicas y sin fines de lucro se encuentran relaciones más acusadas entre TIC y productividad que en las de los sectores manufacturero y financiero.

\section{Conclusiones}

La evidencia empírica registrada en muchos países de la OCDE desde los años 90 permite afirmar que la paradoja de la productividad a nivel de empresa no parece ser tal. Desde mediados de los 90, y a pesar de las limitaciones en las muestras y de la existencia de importantes sesgos, la literatura pone de manifiesto que las inversiones en TIC contribuyen al crecimiento de la productividad del trabajo a través de los efectos directos derivados de la intensificación del capital (capital deepening), así como al de la productividad total de los factores, de trascendental importancia para el crecimiento a largo plazo de cualquier país.

La evidencia refleja que los efectos de los impactos de las TIC son distintos según nos refiramos al corto o al largo plazo. Por una parte, a medida que se va disponiendo de muestras más amplias y se mejoran las técnicas y los modelos, y por otra, a medida que las empresas van aprendiendo y asimilando las inversiones en TIC realizadas, la literatura va recogiendo el análisis de los impactos indirectos de las TIC, de las relaciones entre estas inversiones y los cambios complementarios, asociados principalmente al capital organizacional y humano y de sus efectos sobre el crecimiento de la productividad total de los factores.

Si bien los impactos de las TIC sobre la productividad aparecen asociados en el largo plazo a cambios organizacionales, la evidencia disponible no es concluyente con respecto a la naturaleza de esas relaciones. Los resultados siguen estando condicionados por una amplia variedad de factores, entre los que destacan las dificultades de medición tanto en los aspectos relacionados con la adopción de las TIC como a los relativos a la incorporación en los modelos de las variables organizacionales y a la consideración y medición de los aspectos intangibles. A lo anterior hay que añadir las dificultades para determinar la dirección de la causalidad entre los aspectos tecnoló- 
gicos y organizativos. Por otra parte, los resultados dependen de las características y tamaño de las empresas, de su estrategia organizativa y de su capacidad de innovación, entre otros factores. También dependen de cómo se usen las tecnologías, del papel del capital humano — su cualificación, estructura y organización dentro de la empresa- y del grado en el que se implanten distintos tipos de tecnologías, al jugar las redes un importante papel en el aumento de la productividad.

Una amplia mayoría de los estudios ha estado sesgada hacia empresas grandes en las que existía una inversión previa en TIC. Es probable que en empresas en las que se vaya asimilando la inversión en ordenadores se puedan registrar menores incrementos de la productividad en el futuro, como algunos trabajos referidos al caso español han puesto ya de manifiesto (López Sánchez, 2004).

La extensión del estudio no nos permite hacer un análisis por áreas geográficas, sin embargo de cara a futuras revisiones de la literatura sería interesante situar la evidencia disponible a nivel empresa para distintos países de la OCDE en el contexto de la evolución de la productividad a nivel agregado en cada uno de ellos. En el caso español, habida cuenta de la desfavorable evolución del crecimiento de la productividad a nivel agregado en la década de los 90 y de que las mejoras en dicha evolución a partir del 2000 se asocian a sectores intensivos en TIC (Más y Quesada, 2006), el estudio de los impactos de las inversiones en TIC sobre la productividad en el tejido empresarial adquiere una especial relevancia. La evidencia demuestra los efectos de las inversiones TIC en la mejora de la productividad de las empresas españolas. Sin embargo, la literatura disponible permite avanzar muy lentamente en el análisis, especialmente por lo que se refiere a las PYMES. Todavía son numerosos los ámbitos en los que se requiere continuar progresando en la investigación para poder extraer conclusiones sobre los impactos y el fin de la paradoja a nivel de empresa en el caso español.

Entre ellos, cabe mencionar la necesidad de mejorar la disponibilidad de datos y superar las carencias del sistema estadístico nacional, así como la necesidad de solventar problemas relacionados con la medición de los inputs y outputs tecnológicos. Sería desaeable ampliar el número de estudios sobre el impacto del cambio organizacional asociado a las TIC y sobre la medición de las inversiones en intangibles. Igualmente sería conveniente disponer de estudios que abarquen nuevos sectores, especialmente dentro del sector servicios. También se requieren nuevas investigaciones que aborden con detenimiento las diferencias en los impactos según tipos de empresas y tecnologías, estudios que contemplen un ámbito temporal más amplio y, cómo no, trabajos que contribuyan a determinar el sentido de la causalidad entre las inversiones y uso de las TIC y los cambios organizacionales y el papel del capital humano. 
Aparte de las cuestiones metodológicas y de disponibilidad de datos, hay que tener en cuenta que los niveles de inversión en estas tecnologías son aún bajos en muchas empresas españolas y que el elevado peso de actividades tradicionales o de bajo valor añadido puede dificultar la adopción de las TIC. Por otra parte, sin embargo, cabe esperar que en un futuro y a medida que aumente la inversión en estas tecnologías y su uso, se puedan registrar incrementos de productividad derivados de los efectos directos de las TIC. En el largo plazo serán los efectos indirectos de dichas inversiones, asociados a cambios organizativos y actividades de innovación, a la cualificación del capital humano y a la variedad en la inversión y uso de distinto tipo de TIC, los factores que podrían explicar aumentos de productividad en las empresas españolas.

\section{REFERENCIAS BIBLIOGRÁFICAS}

Arnal, E., Ок, W.; Torres, R. (2001) «Knowledge, Work Organisations and Economic Growth», Occasional Papers, 50, OECD, París.

Arvanitis, S. (2005) «Computerization, Workplace Organization, Skilled Labour and Firm Productivity: Evidence For the Swiss Business Sector», Economics of Innovation and New Technology, XIV (4) 2005: 225-249.

Atrostic, B.K.; NGuYen, S. (2002) Computer Networks and U.S. Manufacturing Plant Productivity: New Evidence from the CNUS Data, enero, U.S. Bureau of the Census CES.

- (2004) How Businesses Use Information Technology: Insights for Measuring Capital and Productivity, junio, U.S. Bureau of the Census CES.

Autor, D.; Katz, L.; Krueger, A. (1998) «Computing Inequality: Have Computers Changed the Labor Market?», Quarterly Journal of Economics, CXIII (4) 1998: 1169-1213.

Autor, D.; Levy, F.; Murnane, R. (2003) «The Skill Content of Recent Technological Change: an Empirical Exploration», Quarterly Journal of Economics, CXVIII (4) 2003: 1279-1333.

Baldwin, J.R.; SAbourin, D.; SMIth, D. (2004) «Firm Performance in the Canadian Food Processing Sector: the Interaction between ICT, Advanced Technology Use and Human Resource Competencies», en: The Economic Impact of ICT. Measurement, Evidence and Implication, París: OECD.

Bartelsman, E.J.; Doms M. (2000) «Understanding Productivity: Lessons from Longitudinal Microdata», Journal of Economic Literature, XXXVIII 2000: 569-594.

Bartelsman, E.J.; Leeuwen, G.; Nieuwenhuissen, H. R. (1996) «Advanced Manufacturing Technology and Firm Performance in the Netherlands», Netherlands Official Statistics, XI 1996: 40-51.

Barua, A.; Kriebel, C. H.; Mukhopadhyay, T. (1995) «Information Technologies and Business Value: an Analytic and Empirical Investigation», Information Systems Research, VI (1) 1995: 3-23. 
Bayo-Moriones, J.A.; Billón-Currás, M.; Lera-López, F. (2007) «Skills, Technology and Organisational Innovations in Spanish Firms», International Journal of Manpower (en prensa).

Bayo-Moriones, J.A.; Lera-López, F. (2007) «A Firm-Level Analysis of Determinants of ICT Adoption in Spain», Technovation, XXVII (6-7) 2007: 352-366.

BertscheK, I.; Kaiser, U. (2004) «Productivity Effects of Organizational Change: Microeconometric Evidence», Management Science, L (3) 2004: 394-404.

Biscourp, P.; Crépon, B.; Heckel, T.; Riedinger, N. (2002) How Do Firms Respond to Cheaper Computers? Microeconometric Evidence for France Based on a Production Function Approach. Abril, G2002/05, Paris: INSEE.

Black, S. E.; Lynch, L. M. (1996) «Human Capital Investments and Productivity», American Economic Review, LXXXVI (2) 1996: 263-267.

- (2001) «How to Compete: the Impact of Workplace Practices and Information Technology on Productivity», Review of Economics and statistics, LXXXIII 2001: 435-445.

- (2004) «What's Driving the New Economy?: The Benefits of Workplace Innovation», The Economic Journal, CXIV (493) 2004: 97-116.

Bresnahan, T. F.; Trajtenberg, M. (1995) «General Purpose Technologies and Aggregate Growth», Journal of Econometrics, LV 1995: 83-108.

Bresnahan, T. F.; Brynjolfsson, E.; Hitt, L.M. (2002) «Information Technology, Workplace Organization and the Demand for Skilled Labor: Firm Level Comments Firm Level Evidence», Quarterly Journal of Econonics, CXVII (1) 2002: 339-376.

Brynjolfsson, E. (1993) «The Productivity Paradox of Information Technology», Communications of ACM, XXXVI (12) 1993: 66-77.

BRYnJolfsson, E.; Hitt, L.M. (1995) «Information Technology as a Factor of Production: The Role of Differences among Firms», Economics of Innovation and New Technology, III (3) 1995: 183-199.

- (1996) «Paradox Lost? Firm-Level Evidence on the Returns to Information Systems Spending», Management Science, XLII (4) 1996: 541-558.

- (1998) «Beyond the Productivity Paradox: Computers Are the Catalyst for Bigger Changes», Communications of ACM, XLI (8) 1998: 49-55.

- (2000) «Beyond Computation: Information Technology, Organizational Transformation and Business Practices», Journal of Economic Perspectives, XIV (4) 2000: 23-48.

- (2003) «Computing Productivity: Firm-Level Evidence», Review of Economics \& Statistics, LXXXV (4) 2003: 793- 809.

BRYnJolfsson, E.; Hitt, L.M.; YANG, S. (2002) «Intangible Assets: Computers and Organizational Capital», Brooking Papers on Economic Activity, MMII (1) 2002: 137-181.

Brynjolfsson, E.; YANG, S. (1996) «Information Technology and Productivity: a Review of the Literature», Advances in Computers, XLIII 1996: 179-214.

Brynson, K.; Ko, M. (2004) «Exploring the Relationship Between Information Technology Investments and Firm Performance Using Regression Splines Analysis», Information \& Management, XLII 2004: 1-13. 
Clayton, T.; Criscuolo, C.; Goodridge, P.; Waldron, K. (2004) «Enterprise e-commerce: Measurement and Impact», en: The Economic Impact of ICT. Measurement, Evidence and Implications, Paris, OECD: 241-260.

Colecchia, A.; Schreyer, P. (2002) «ICT Investment and Economic Growth in the 1990s: Is the United States a Unique Case? A Comparative Study of Nine OECD Countries», Review of Economic Dynamics, V (2) 2002: 408-442.

DANs, E. (2001) «IT Investment in Small and Medium Enterprises: Paradoxically Productive?», The Electronic Journal of Information Systems Evaluation, IV (1) 2001: 1-25.

Daveri, F. (2002) «The New Economy in Europe, 1992-2001», Oxford Review of Economic Policy, XVIII (3) 2002: 345-362.

Dedrick, J.; Gurbaxani, V.; Kraemer, K, L. (2003) «Information Technology and Economic Performance: a Critical Review of the Empirical Evidence», ACM Computing Surveys, XXXV (1) 2003: 1-28.

Dehning y Richardson (2002) «Returns on Investments in Information Technology: A Research Synthesis», Journal of Information Systems, XVI (1) 2002: 7-30.

Devaraj S.; Kohl, R. (2003) «Performance Impacts of Information Technology: is Actual Usage the Missing Link?», Management Science, XLIV (3) 2003: 273-289.

Dewan, S. M.; Min, C. K. (1997) «Substitution of Information Technology for other Factors of Production: a Firm Level Analysis», Management Science, XLIII (12) 1997: 1660-1675.

Dewan, S. M.; Steven C. (1998) «Firm Characteristics and Investments in Information Technology: Scale and Scope Effects», Information Systems Research, IX (3) 1998: 219-233.

Dewett, T.; Jones, G.R. (2001) «The Role of Information Technology in the Organization: a Review, Model, and Assessment», Journal of Management, XXVII 2001: 313-346.

DMR Consulting; SEDISI (2003) Las Tecnologías de la Sociedad de la Información en la Empresa Española 2000, Madrid, SEDISI y DMR Consulting.

DMR Consulting; AETIC (2004) Las Tecnologías de la Sociedad de la Información en la Empresa Española 2003, Madrid, AETIC y DMR Consulting.

Doms, M.; Dunne, T.; Troske, K.R. (1997) «Workers, Wages and Technology», Quarterly Journal of Economics, CXII (1) 1997: 253-290.

Dunne, T.; Schmitz, J. (1995) «Wages, Employment Structure and Employer Size- Wage Premia: their Relationship to Advanced-Technology Usage at US Manufacturing Establishments», Economica, LXII (254) 1995: 89-107.

Entorf, H.; Kramarz, F. (1998) «The Impact of New Technologies on Wages: Lessons from Matching Panels on Employees and on their Firms», Economic Innovation and New Technology, V 1998: 165-197.

Estrada, A.; Pons, A.; VAllés, J. (2006) «La Productividad de la Economía Española: una Perspectiva Internacional, Información Comercial Española. Revista de Economía, DCCCXXIX 2006: 7-25.

Francalanci, C.; Galal, H. (1998) «Information Technology and Worker Composition: Determinants of Productivity in the Life Insurance Industry», MIS Quarterly, XXII (2) 1998: 227-241. 
Fuentelsaz, L.; Maicas, J.P; Polo, Y. (2005) «Hacia una Gestión Eficiente de las Tecnologías de la Información y las Comunicaciones», Universia Business Review, VI ( $2^{\circ}$ trimestre) 2005: 40-53.

Gargallo, A. Galve, C.; (2004) «Impacto de las Tecnologías de la Información en la Productividad de las Empresas Españolas», DTECONZ 2004-05 C., Universidad de Zaragoza. Facultad de CC. Económicas y Empresariales.

GAPTEL (2004) Productividad, Crecimiento Económico y TIC, Marzo, Madrid, Red.es.

Gilchrist, S.; Gurbaxani, V.; Town, R. (2001) «PCs and the Productivity Revolution», Working paper, Irvine, University of California, Center for Research on Information Technology and Organizations.

Gimeno (2006) «Las Tecnologías de la Información y la Comunicación en las PYMES y otros Avatares», Economía Industrial, CCCLX 2006: 77- 91

Gordon, R. J. (2000) «Does the «New Economy» Measure up to the Great Inventions of the Past?», Journal of Economic Perspectives, IV (14) 2000: 49-74.

Goss, E. (2001) «The Internet's Contribution to U.S. Productivity Growth», Bussines Economics, XXXVI (4) 2001:32-42.

Greenan, N.; Mairesse, J.; Topiol-Bensaid, A. (2001) «Information Technology and Research and Development Impacts on Productivity and Skills: Looking for Correlations on French Firm Level Data», en: Pohjola, M. (ed.) Information Technology, Productivity, and Economic Growth: International Evidence and Implications for Economic Development. Cambridge, Oxford University Press: 119-148.

Gretton, P.; Gali, J.; Parham, D. (2002) Uptake and Impacts of ICT in the Australian Economy: Evidence from Aggregate, Sectoral and Firm Levels, OECD Workshop on ICT and Business Performance, Canberra, OECD.

Hempell, T. (2005) «Does Experience Matter? Innovations and the Productivity of Information and Communication Technologies in German Services», Economics of Innovation and New Technology, XIV (4) 2005: 277-303.

Hernando, I.; NúÑEz, S. (2004) «The Contribution of ICT to Economic Activity: a Growth Accounting Exercise with Spanish Firm-Level Data», Investigaciones Económicas, XXVIII (2) 2004: 315-348.

Hitt, L.M.; Brynjolfsson, E. (1996) «Productivity, Business Profitability, and Consumer Surplus: Three Different Measures of Information Technology Value», MIS Quarterly, XX (2) 1996: 121-142.

Hollenstein, H. (2004) «The Decision to Adopt Information and Communication Technologies (ICT) Firm-Level Evidence for Switzerland», en: OECD, The Economic Impact of ICT. Measurement, evidence and implications, Paris, OECD: 37-60.

Jorgenson, D.W. (2001) «Information Technology and the US Economy», American Economic Review, XCI (1) 2001: 1-32.

Kohl, R.; DevaraJ, S. (2003) «Measuring Information Technology Payoff: a Meta-Analysis of Structural Variables in Firm-Level Empirical Research», Information System Research, XIV (2) 2003:127-145. 
Krueger, A.B. (1993) «How Computers Have Changed the Wage Structure: Evidence from Microdata, 1984-1989», The Quarterly Journal of Economics, CVIII (1) 1993: 33-60.

Lehr, B.; Lichtenberg, F. (1999) «Information Technology and its Impact on Productivity: Firm-Level Evidence from Government and Private Data Sources», Canadian Journal of Economics, XXXII (2) 1999: 335-362.

Liснт, G.; Мосн, D. (1999) «Innovation and Information Technology in Services», Canadian Journal of Economics-Revue Canadienne d'Economique, XXXII (2) 1999: 363-383.

Lichtenberg, F. (1995) «The Output Contributions of Computer Equipment and Personnel: a firm Level Analysis», Economics of Innovation and New Technology, III 1995: 201-217.

Llanos Matea, M. (2006) «Los Problemas de Medición por Cambios de Calidad en las TIC: Evidencia para España», Economía Industrial, CCCLX 20006: 107-115.

López SÁnchez, J.I. (2004) «¿Pueden las Tecnologías de la Información Mejorar la Productividad?», Universia Business Review, I primer trimestre 2004: 82-95.

López Sánchez, J.J.; Minguela, B.; Rodríguez, A.; Sandulli, F.D. (2003) «Uso de Internet y Paradoja de la Productividad: el Caso de la Empresa Española», XII Congreso Nacional ACEDE, Salamanca.

Loveman, G. W. (1994) «An Assessment of the Productivity Impact of Information Technologies», en: Allen, T.J.; Scott, M.S. Information Technology and the Corporation of the 1990s: Research Studies, Cambridge, U.K., Eds. Oxford University Press: 84-110.

Mahmood, M.A, SzewczaK (1998) Measuring Information Technology Investment Payoff: Contemporary approaches, Pennsylvania, Idea Group Publishing: 486-490.

Mas, M.; Quesada, J. (dirs.) (2005a): Las Nuevas Tecnologías y el Crecimiento Económico en España, Bilbao: Fundación BBVA.

- (2005b): «ICT and Economic Growth: A Quantification of Productivity Growth in Spain 1985-2002», OCDE Statistics Working Paper, STD/DOC (2005)4, 17 Agosto.

- (2006): «The Role of ICT in the Spanish Productivity Slowdown», Documento de Trabajo n. 5, Bilbao: Fundación BBVA,

Matteucci, N.; O’Mahony, M.; Robinson, C.; Zwick, T. (2005) Productivity Workplace Performance and ICT: Industry and Firm-Level Evidence for Europe and the U.S.», Scottish Journal of Political Economy, LII (3) 2005: 359-386.

Melville, N.; Kraemer, K.L.; Gurbaxani, V. (2004) «Review: Information Technology and Organizational Performance: an Integrative Model of IT Business Value», MIS Quarterly, XXVIII (22) 2004: 283-322.

Menon, N.; Lee, B.; Eldenburg, L. (2000) «Productivity of Information System in the Healthcare Industry», Information Systems Research, XI (1) 2000: 83-92.

Milgrom, P.; Roberts, J. (1990) «The Economics of Modern Manufacturing: Technology, Strategy and Organization», American Economic Review, LXXX (6) 1990: 511-528.

Miller, E.; Rice, A. (1967) Systems of Organization: The Control of Task and Sentient Boundaries, London, Tavistock. 
Nordhaus, W. D. (2001) Productivity Growth and the New Economy, NBER Working Paper 8096, [On-line]. Disponible en URL: http://www.nber.org/papers/w8096.

OCDE (2001) Measuring Productivity, OECD Manual, París, OCDE.

- (2003) Information and Communications Technologies. ICT and Economic Growth. Evidence from OECD Countries, Industries and Firms, París, OCDE.

- (2004) The Economic Impact of ICT Measurement, Evidence and Implications, París, OCDE.

Oliner, S.D.; Sichel, D.E. (2000) «The Resurgence of Growth in the Late 1990's: Is Information Technology the Story?», Journal of Economic Perspectives, IV (14) 2000:3-22

Ontiveros, E.; Manzano, D.; Rodríguez, I. (2004) Tecnologías de la Información y las Comunicaciones (TIC), Crecimiento Económico y Actividad Empresarial, Madrid, Círculo de Empresarios.

Oz, E. (2005) «Information Technology Productivity: in Search of a Definite Observation», Information and Management, XLII (6) 2005: 789-798.

Pérez, M.; Martínez, A.; de Luis, P.; Vela, M.J. (2006) «Las TIC en las PYMES: Estudio de Resultados y Factores de Adopción», Economía Industrial, CCCLX 2006: 93-105.

Pilat, D. (2004) The ICT Productivity Paradox: Insights from Micro Data, OECD Economic Studies, nº 38, 2004/1.París, OCDE.

- (2005) Spain's Productivity Performance in International Perspective, documento preparado para el OECD Workshop on Productivity, Madrid, 17-19 octubre.

Pilat, D.; Wyckoff, A. (2005) «The Impacts of ICT on Economic Performance: An International Comparison at Three Levels of Analysis» en: Transforming Enterprise: The Economic and Social Implications of Information Technology, Cambridge and London: MIT Press: 77-109.

Prasad, B.; Harker, P. (1997) Examining the Contribution of Information Technology Toward Productivity and Profitability in U.S. Retail Banking, Working Paper, n97-09, Pennsylvania, The Wharton School, University of Pennsylvania.

Roach, S. S. (1991) «Services Under Siege: the Restructuring Imperative». Harvard Business Review, XXXIX (2) 1991: 82-92.

Sanaú, J.; Barcenilla, S.; LóPez-Pueyo, C. (2006) «Productividad Total de los Factores y Capital Tecnológico: un Análisis Comparado», Información Comercial Española. Revista de Economía, DCCCXXIX 2006: 145-163.

SAnjurjo, E. (2003) Contribución de las TIC al Crecimiento Económico. Estimación Basada en Datos Españoles a Nivel de Empresa. Tesis Doctoral, Master en Economía Industrial, UC3M, Madrid.

STIROH, K.J. (2002) «Information Technology and the U.S. Productivity Revival: What Do the Industry Data Say?», American Economic Review, XCII (5) 2002: 1559-1576.

Strassmann, P. A. (1990) The Business Value of Computers: an Executive's Guide, New Canaan, $C T$, The Information Economic Press.

Timmer, M.P.; van Arky, B. (2005) «Does Information and Communication Technology Drive 
EU-US Productivity Growth Differentials?», Oxford Economic Papers, LVII (4) 2005: 693-716.

Uriel, E. (2006) Uriel, E. (2006) La Medición de la Productividad en España. Ponencia presentada en el Seminario internacional Capitalización y crecimiento sostenido: Experiencias internacionales, análisis y política, Buenos Aires, 4-5 de diciembre de 2006, Fundación BBVA, CEPAL, OECD, BBVA Fundación Banco Francés, IVIE.

VILASECA, J. (dir.) (2003) Las TIC y las Transformaciones de la Empresa Catalana, Barcelona, Observatori Nova Economia.

Webster, J.; Watson, R. (2002) «Analyzing the Past to Prepare for the Future: Writing a Literature Review». MIS Quarterly, XXVI (2) 2003: xiii-xxiii.

Zwick, T. (2003) «The Impact of ICT Investment on Establishment Productivity», National Institute Economic Review, CLXXXIV April 2003: 70-81. 